\title{
SPONTANEOUS CP VIOLATION: ALTERNATIVE TO THE STANDARD MODEL
}

\author{
Paul H. Frampton \\ Institute of Field Physics, \\ Department of Physics and Astronomy, \\ University of North Carolina, CB \#3255, Chapel Hill. NC 27599-3255. \\ E-mail: frampton@physics unc,edu
}

\begin{abstract}
We propose a model of soft $\mathrm{CP}$ violation that evades the strong $\mathrm{CP}$ problem and can describe observed $\mathrm{CP}$ violation in the neutral kaon sector, both direct and indirect. Our model requires two "duark" mesons carrying quark number two that have complex (CP-violating) bare masses and are coupled to quark pairs. Aside from the existence of these potentially observable new particles with masses of several hundred $\mathrm{GeV}$, we predict a flat unitarity triangle (i.e., no observable direct $\mathrm{CP}$ violation in the $B$-meson sector) and a possibly anomalous branching ratio for the decay mode $K^{+} \rightarrow \pi^{+}+\bar{\nu} \nu$.
\end{abstract}

\section{Introduction}

I am happy to be invited at this Inaugural Conference of the Michigan Center for Theoretical Physics, and to wish for the Center a glorious and distinguished future.

The standard model of particle physics involves three fermion families and one Higgs doublet. Within this model, $\mathrm{CP}$ violation can manifest itself in just

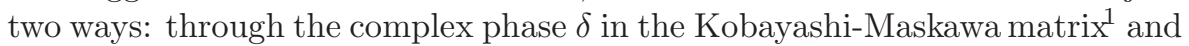
through the coefficient $\bar{\theta}$ of the Chern-Simons term目. The complex Yukawa couplings of the Higgs boson can contribute to of both these parameters, so that they would be expected to be comparable in magnitude. However, the standard model requires $|\delta| \sim 1$ to describe $\mathrm{CP}$ violation for neutral kaons, yet it also requires $|\bar{\theta}| \leq 1.5 \times 10^{-10}$ lest observable nuclear electric dipole moments be generated. This dramatic departure from naturality is the gist of the strong CP problem, whose solution is a primary goal of this paper which is based on work with Glashow and Yoshikaw 1 .

There are other reasons to consider alternatives to the standard description of $\mathrm{CP}$ violation: Recall that the $\mathrm{CP}$-violation implicit in the standard model does not seem to be sufficient to implement the prescient idea of Sakhargy through which the baryonic asymmetry of the universe may be generated. 
Furthermore it would seem useful to have other models in hand in the event that experiments now being carried out do not confirm the standard-model prediction that $\sin 2 \beta=0.7 \pm 0.2$, where $\beta$ is one of the vertex angles of the unitarity triangle. In this connection, we note that unofficial averages of various experimental results, as well as indirect theoretical arguments based on other available dat 6 , disfavor but do not yet exclude our prediction that $\sin 2 \beta \simeq 0$.

Several simple fixes to the strong CP problem have been suggested. The simplest of these, a massless up quark or an invisible axion, are all but excluded by observation and theoretical analysis. In more elaborate models, CP is assumed to be violated spontaneously (which usually leads to unacceptable domain walls), or softly 8.10 . In the latter models, various new heavy fermions and heavy bosons are introduced with $\mathrm{CP}$ conservation imposed on all dimension-4 terms in the Lagrangian, but not on the lower-dimension bare mass terms of the new particles. Most of these models have been excluded by experiment: they are superweak mimics that cannot reproduce the observed value of $\left(\epsilon^{\prime} / \epsilon\right)_{K}$.

\section{The Duark Model}

Our model of soft CP violation is simpler than its predecessors in that it requires new bosons, but no new fermions. Let us begin with the Lagrangian for a variant of the standard model where: (a) CP invariance is imposed on all dimension-four terms; and (b) two Higgs multiplets are introduced. The latter hypothesis requires clarification. A discrete symmetry $\mathcal{D}$ must be imposed on the Lagrangian to ensure that one Higgs multiplet $H^{u}$ gives rise to upquark masses, while the other $H^{d}$ gives rise to down-quark masses. A suitable choice for $\mathcal{D}$ is an operation under which all right-handed quarks and $H^{u}$ are odd, with all other fields even. Note that $\mathcal{D}$ invariance forbids Higgs mass terms proportional to $H^{u \dagger} H^{d}$ which (if complex) would directly contribute to Arg Det $M$. At this point in the explication of our model the Lagrangian is entirely $\mathrm{CP}$ conserving, with $\bar{\theta}=0$ and a real orthogonal KM matrix 11 .

The essential extension of the above-described Lagrangian consists of two spinless bosons $\phi^{(a)} \quad(a=1,2)$ that carry quark number two (or baryon number $\frac{2}{3}$ ) and couple to quark pairs. We assume that each of the $\phi^{(a)}$ is a color antitriplet and weak $S U(2)$ singlet with electric charge $Q=\frac{1}{3}$. These particles are hereafter referred to as "duarks." To specify the duark couplings, we denote the left-handed quark doublets by $\Psi_{L}$ where: 


$$
\Psi_{L}=\left(\begin{array}{c}
u_{L} \\
V d_{L}
\end{array}\right)
$$

with color, flavor, and Dirac indices suppressed. The $u_{L}$ and $d_{L}$ are left-handed quarks in the basis in which the tree-level mass matrices are diagonal, and $V$ is the tree-level KM matrix, which is real and orthogonal by hypothesis. The duark couplings to quarks may be written:

$$
f \phi_{i}^{(a)} \epsilon_{i j k} \epsilon_{b c} o_{n m}^{(a)}\left(\tilde{\Psi}_{L}^{j b n}\left(i \gamma_{0} \gamma_{2}\right) \Psi_{L}^{k c m}\right)+\text { h.c. }
$$

where the tilde transposes the Dirac indices and the real antisymmetric matrix $i \gamma_{0} \gamma_{2}$ produces a Lorentz scalar. Indices $a=1,2$ label the duarks, indices $i, j, k$ label colors, indices $b, c$ label weak isospin, and indices $n, m$ label the flavors of the quark doublets. The subscripted $\epsilon$ 's are the usual invariant antisymmetric matrices. The constant $f$ sets the overall scale of the couplings. The $o_{n, m}^{(a)}$ are $3 \times 3$ real symmetric matrices in flavor space. All their entries are assumed to be of order unity in lieu of specific theoretical insight. In brief, $\phi^{(a)}$ couples to the quark pair $\left(u_{L n}, d_{L m}\right)$ with coupling strength $f o_{n l}^{a} V_{l m}$. These duarkquark couplings are necessarily flavor symmetric in the weak-isospin basis, but they depart from symmetry in the mass-eigenstate basis we use.

The need for two Higgs bosons now becomes apparent. We have required duarks to couple to pairs of left-handed quarks, but not to pairs of right-handed quarks. This is a proper and renormalizable condition if and only if we take the $\phi^{(a)}$ to be even under the discrete symmetry $\mathcal{D}$. (The other choice of $\mathcal{D}$-parity for duarks leads to a model with a strong CP problem.) We note in passing that $\mathcal{D}$ is broken spontaneously along with $S U(2) \times U(1)$, so that finite duark couplings to right-handed quarks arise at one loop. These are suppressed by a product of Higgs coupling constants and by a canonical factor of $(4 \pi)^{-2}$ and are small enough to have no effect on our subsequent arguments.

Aside from their quartic self couplings (which play no role here), the duarks have bare masses (specified by $\mathcal{M}^{2}$ ) and quartic couplings to the Higgs bosons:

$$
\phi^{(a) \dagger} \phi^{(b)} \mathcal{M}_{(a b)}^{2}+f^{2} \phi^{(a) \dagger} \phi^{(b)}\left\{\alpha_{(a b)}^{u} H^{u \dagger} H^{u}+\alpha_{(a b)}^{d} H^{d \dagger} H^{d}\right\}
$$

The latter interaction is taken to have a coupling strength of order $f^{2}$, a hypothesis both suggested by and compatible with the assumed strength of the duark Yukawa couplings to quarks. The matrices $\alpha^{u, d}$ are real and symmetric (CP conserving) with entries, once again, assumed to be of order unity. The bare masses of the duarks are described by the complex Hermitean matrix $\mathcal{M}$. 
Indeed, the sole source of $\mathrm{CP}$ violation in our model lies in the bare masses of the duarks.

The duark mass eigenstates $\Phi^{(a)}$ are the eigenvectors of their complete mass matrix $\mathcal{M}^{\prime 2}$, which is the sum of $\mathcal{M}^{2}$ and small additional terms obtained by replacing the Higgs bosons by their vev's in Eq. 3. We denote these eigenstates by $\Phi=u^{\dagger} \phi$, where $u$ is a $2 \times 2$ unitary unimodular matrix. Both mass eigenvalues $M^{(a)}$ and their difference are assumed to be of comparable magnitude and are denoted by $M$. This crude approximation (and the other simplifications we have made) compel us to remind the reader that the estimates we shall offer for duark masses and couplings are merely order of magnitude estimates.

We rewrite the duark couplings given by Eq. 2 in terms of these mass eigenstates. That is, we replace $\phi^{(a)}$ by $\Phi^{(a)}$ and the real symmetric matrices $o^{(a)}$ by the complex symmetric matrices $O^{(a)} \equiv o^{(b)} u_{b a}$. In this manner, the $\mathrm{CP}$ violation is transferred from the mass terms of the duarks to their couplings with quarks. The complex (CP violating) phases of these couplings are assumed to be of order unity.

\section{CP Violation in The Neutral Kaon Sector}

As in any model of soft $\mathrm{CP}$ violation, the box diagram shown in Fig. 1, with two incoming strange quarks and two exiting down quarks, generates an effective 4 -fermion coupling of the form $\left(\bar{d}_{L} \gamma^{\mu} s_{L}\right)\left(\bar{d}_{L} \gamma_{\mu} s_{L}\right)$ which must be wholly responsible for indirect $\mathrm{CP}$ violation in the neutral kaon sector. 


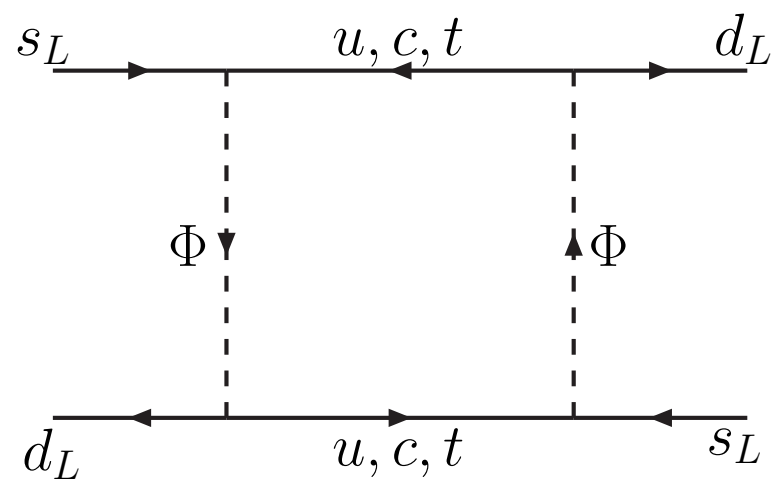

Fig. 1. Box diagram

Setting its coefficient equal to the experimentally determined value of $\epsilon \Delta m_{K}$, one obtains 10 the constraint:

$$
\frac{\alpha_{f}}{M} \approx 2 \times 10^{-8} \mathrm{GeV}^{-1}
$$

with $\alpha_{f}=f^{2} / 4 \pi$ and $M$ an estimate of the duark mass scale.

We turn to the question direct $\mathrm{CP}$ violation in the kaon sector, such as discussed in 12,110 . Here our model differs radically from its predecessor: the exchange of a duark between two quark pairs, as shown in Fig. 2, generates small and non-conventional four-fermion couplings that contribute to non-leptonic decays. 


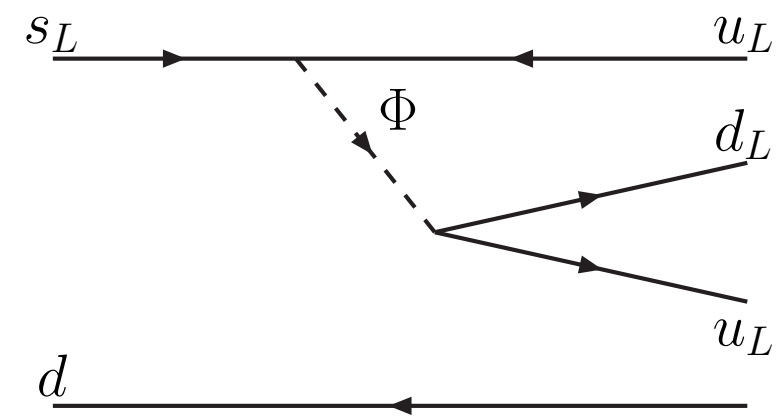

Fig. 2 Tree level contribution to direct $\mathrm{CP}$ violation

These are of no significant observable consequence, except for the case of neutral kaons where the relevant term $\left(\bar{d}_{L} \gamma_{\mu} u_{L}\right)\left(\bar{u}_{L} \gamma^{\mu} s_{L}\right)$ is purely $\Delta I=\frac{1}{2}$, has magnitude $\sim f^{2} / M^{2}$ with a large but unknown complex phase $\eta$. (It also has an unconventional color structure.) This term contributes comparably to the 2-pion decays of both $K_{L}$ and $K_{S}$, so that the overall decay amplitudes will satisfy:

$$
A_{2} /\left.A_{0}\right|_{S}=\omega(1-\zeta \cos \eta-i \epsilon \zeta \sin \eta)
$$

and

$$
A_{2} /\left.A_{0}\right|_{L}=\omega(1-\zeta \cos \eta-i(\zeta / \epsilon) \sin \eta),
$$

where $\omega \equiv A_{2} /\left.A_{0}\right|_{S} \simeq 1 / 22, \zeta$ is the ratio of the strength of the duark exchange amplitude to that arising from $W$ exchange, and $\eta$ is its unknown phase:

$$
\zeta \approx \frac{f^{2}}{M^{2}}\left(\sqrt{8} G_{F} \sin \theta_{c}\right)^{-1}
$$

with $\theta_{c}$ is the Cabibbo angle. Here we ignore the difference in color structure of the two amplitudes. 
From these results (and the known relation among pion phase shifts, $\delta_{2}-$ $\left.\delta_{0} \approx-\pi / 4\right)$ we deduce:

$$
1-6 \frac{\epsilon^{\prime}}{\epsilon} \equiv\left|\frac{\eta^{+-}}{\eta^{00}}\right|^{2}=1 \pm \frac{3 \zeta \omega}{\epsilon}
$$

where we have arbitrarily chosen $\eta= \pm \pi / 2$ for the unknown phase. ¿From this result we obtain

$$
\left|\frac{\epsilon^{\prime}}{\epsilon}\right| \approx\left|\frac{\zeta \omega}{2 \epsilon}\right|
$$

If Eq.(8) is to yield the observed value $\epsilon^{\prime} / \epsilon \simeq 2 \times 10^{-3}$, we must have $\zeta \approx$ $1.8 \times 10^{-4}$. Making use of this result and Eq. 6 , we obtain the following order of magnitude constraint:

$$
\frac{\alpha_{f}}{M^{2}} \approx 10^{-10} \mathrm{GeV}^{-2}
$$

which together with Eq. 4, the constraint from indirect CP violation, yields the estimates

$$
M \approx 200 \mathrm{GeV} \text { and } \alpha_{f} \approx 4 \times 10^{-6} .
$$

It must be emphasized that, because of the assumptions used, Eq.(11) gives only order of magnitude estimates: the mass $\mathrm{M}$ is predicted to be several hundred $\mathrm{GeV}$. We have shown that a correct description of $\mathrm{CP}$ violation in the neutral kaon system can be obtained with duarks of soon-to-be-accessible masses. However, our model requires that duarks are only weakly coupled to quark pairs.

\section{Direct CP Violation in $B$-Meson Decays}

Our model starts off with a real KM matrix and a degenerate unitarity triangle. Radiative corrections will produce a finite imaginary part of the KM matrix, with the leading contribution arising from the Feyman diagram shown in Fig. 3 . 


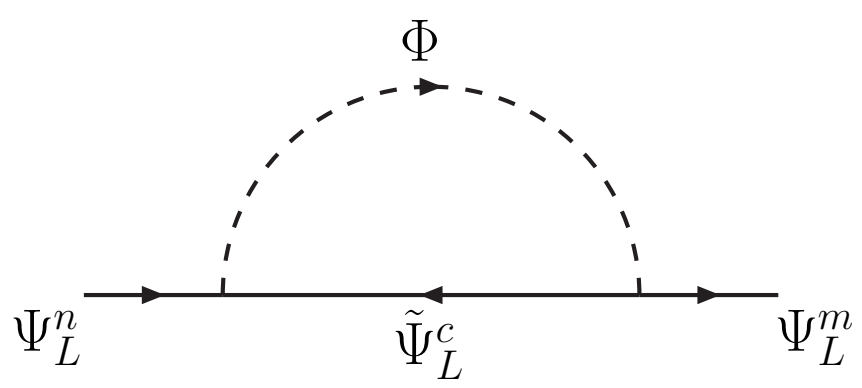

Fig. 3 Self-mass.

The situation here is similar to that in earlier models of soft CP violation, where the area of the unitarity triangle divided by its standard-model value is typically $\sim \alpha_{f} / 4 \pi$, where $\alpha_{f}$ characterizes the couplings of hypothetical new particles to quarks. In our case, this coupling constant is tiny and the unitarity triangle remains experimentally indistinguishable from a straight line. The exchange of a duark between quark pairs, as shown in Fig. 2, does yield a nonstandard contribution to $B$-decay, but one which is three orders of magnitude smaller than that due to $W$ exchange. It does not lead to readily detected effects. We conclude that our model demands a flat unitarity triangle and predicts that there is no observable direct $\mathrm{CP}$ violation in the $B$-meson sector.

\section{The Strong CP Problem}

In our model, as in all models of soft $\mathrm{CP}$ violation, the $\mathrm{QCD} \theta$ parametercorresponding to a dimension- $4 \mathrm{CP}$ violating operator - must vanish. All CPviolating contributions to $\bar{\theta}$ are finite and calculable radiative corrections. We need not consider self-energy diagrams such as that in Fig. 3 because they are associated with Hermitean counter-terms in the Lagrangian and cannot 
contribute to $\bar{\theta}$ to any order in $\alpha_{f}$. Rather, and as further explicated in 10 , we must examine radiative corrections to the Higgs couplings, which is to say, corrections to quark masses $\left(\Delta M_{U}\right.$ and $\left.\Delta M_{D}\right)$ whose contribution to the phase of the determinant of the quark masses is:

$$
\Delta \bar{\theta} \approx \operatorname{Im} \operatorname{Tr}\left(\Delta \mathrm{M}_{\mathrm{D}} \mathrm{M}_{\mathrm{D}}^{-1}\right)+\operatorname{Im} \operatorname{Tr}\left(\Delta \mathrm{M}_{\mathrm{U}} \mathrm{M}_{\mathrm{U}}^{-1}\right)
$$

Earlier models of soft CP violation involve the existence of new heavy particles (both mesons and fermions) which are very large compared to the electroweak scale. In contrast, our new particles (duark mesons) have relatively small masses. Thus, quark masses appearing in the denominators of Feynman integrals contributing to $\Delta M_{U}$ and $\Delta M_{D}$ cannot be ignored. However, this complication is alleviated by the tiny value we have deduced for $\alpha_{f}$. It is sufficiently small that we need examine only those quark mass corrections involving exactly one duark loop. The leading contributions to $\bar{\theta}$ arise from diagrams with one duark loop and one Higgs loop, for which there are two possibilities.

One of the leading contributions to $\bar{\theta}$ in our model arises from the two-loop diagram shown in Fig. 4a.

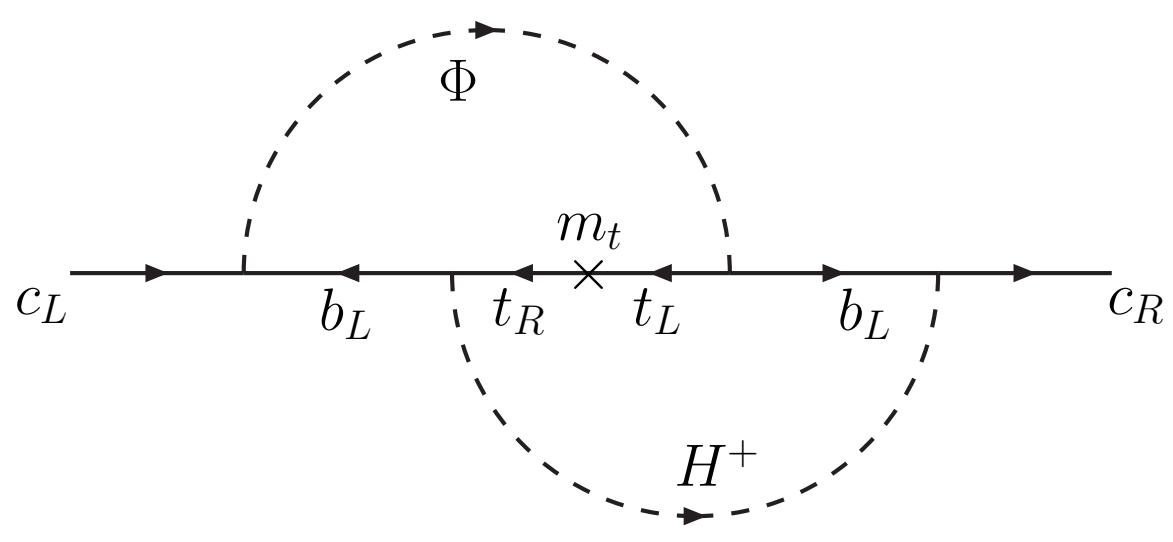

Fig. 4a First 2-loop contribution to $\bar{\theta}$ discussed in the text. 


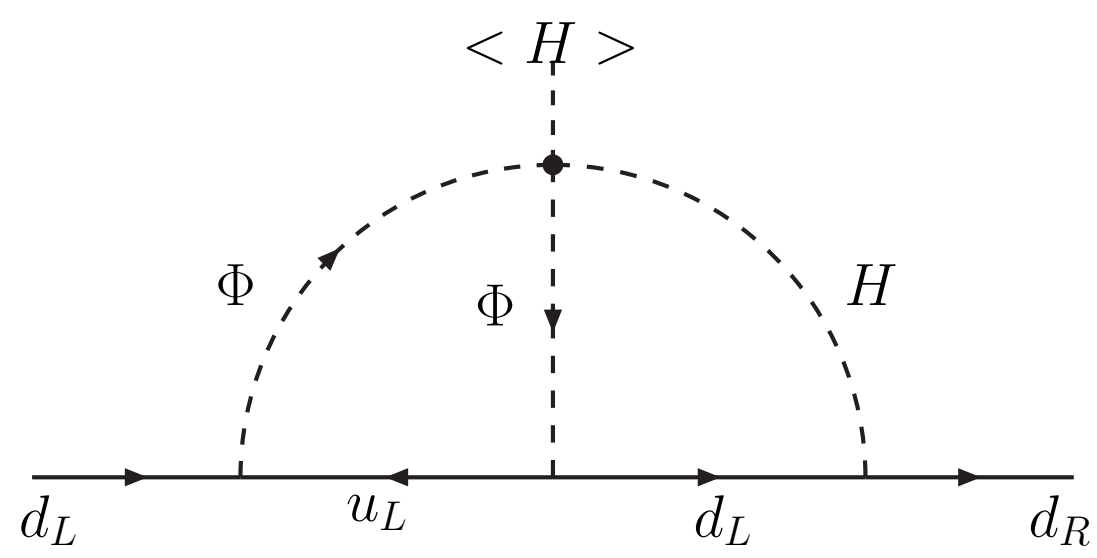

Fig. 4b Second 2-loop contribution to $\bar{\theta}$ as discussed in the text.

The imaginary part of this amplitude tends to zero as the KM matrix $V$ approaches the unit matrix. It also tends to zero if all quark masses (except the one with the mass insertion) are neglected. It follows that these contributions to $\bar{\theta}$ are highly suppressed:

$$
\Delta \bar{\theta} \simeq \frac{\alpha_{f} \lambda^{2}}{(4 \pi)^{3}}\left\{\frac{m_{t}^{2} m_{b}^{2}}{M^{2}\left\langle H^{u}\right\rangle^{2}} \quad \text { or } \quad \frac{m_{b}^{2}}{\left\langle H^{d}\right\rangle^{2}}\right\}
$$

where $\lambda$ is the Wolfenstein parameter whose value is approximately $\sin \theta_{c}$. These radiative corrections yield $\Delta \bar{\theta} \sim 10^{-12}$. The other two-loop contribution to $\bar{\theta}$, shown in Fig. 4b, involves the quartic Higgs-duark coupling of Eq. 3. This radiative correction contributes $\Delta \bar{\theta} \sim\left(\alpha_{f}^{2} / 4 \pi\right)^{2} \simeq 10^{-13}$. It follows that our model is easily compatible with present constraints on the strong $\mathrm{CP}$-violating parameter $\bar{\theta}$. 


\section{Rare Semi-Leptonic Kaon Decays}

Here we consider the rare decay modes $K^{+} \rightarrow \pi^{+} \bar{\nu} \nu$ and $K_{L} \rightarrow \pi^{0} \bar{\nu} \mu$. The first of these has a predicted branching ratio of $(7.9 \pm 3.1) \times 10^{-11} \quad 13$. The observed branching ratio of $\left(15_{-12}^{+34}\right) \times 10^{-11} \quad 14$ agrees with the prediction, but is based on the observation of a single event at the Brookhaven E787 experiment.

In our model, $Z^{0}$ penguin diagrams involving a duark loop contribute to both of these decay modes. The dominant contribution to this diagram (as for the standard-model penguin) involves an intermediate top quark. Thus for the decay $K^{+} \rightarrow \pi^{+} \bar{\nu} \nu$, the ratio of this novel amplitude to the conventional amplitude is given naively by

$$
\zeta^{\prime}=\frac{f^{2}}{M^{2}}\left(\sqrt{8} G_{F} \lambda^{5}\right)^{-1} \simeq 0.1
$$

Of course, this is no better than an order-of-magnitude estimate. In fact, $\zeta^{\prime}$ may be negligible, or it may be of order unity. Thus future measurements of rare semileptonic kaon decays may reveal a significant departure from standard medel predictions. A more careful calculation of the duark contribution (such as 13 in the case of the standard-model result) is certainly premature at present, but will be called for if more precise data becomes available, and if our model of soft $\mathrm{CP}$ violation survives further experimental scrutiny.

\section{Conclusion}

We sketched a model wherein $\mathrm{CP}$ is a softly-broken symmetry of the Lagrangian. Our model yields $|\bar{\theta}|<10^{-12}$ and therefore does not suffer from a strong $\mathrm{CP}$ problem. It is based on a two-Higgs variant of the standard model to which are adjoined two spinless duark mesons that carry baryon number $\frac{2}{3}$ and have $\mathrm{CP}$ violating bare masses. Indirect $\mathrm{CP}$ violation in kaon decay ( $\epsilon$ related) proceeds through a duark box diagram. Unlike other models of soft $\mathrm{CP}$ violation, ours includes direct $\mathrm{CP}$ violation via duark that readily accommodates the observed value of $\epsilon^{\prime}$. It would be interesting to study the incorporation of our model in a larger theoretical scheme such as grand unification or higher dimensions.

${ }^{a}$ Fanciers of supersymmetry may wish to identify anti-duarks with squarks that enjoy $R$-odd couplings that violate baryon number by one. 
Furthermore. our model makes two immediate and decisive predictions: There should be no observable direct $\mathrm{CP}$ violation in the $B$-meson sector. That is, we predict $\sin 2 \beta=0$ to the precision of any currently feasible experiment. Secondly, we predict the existence of duarks with masses that are soon to be experimentally accessible. These particles should be copiously pair-produced at the LHC with picobarn cross sections, and they should decay into assorted quark pairs (tb, td, bc, etc.) with widths well below experimental resolution. Those events involve a $t \bar{t}$ pair and two additional jets should provide a recognizable signal. In addition to these explicit predictions, we find that penguin diagrams involving duarks may contribute significantly to decays of kaons into pions plus $\nu \bar{\nu}$ pairs.

\section{Acknowledgements}

The researches of PHF and TY were supported in part by the US Department of Energy under Grant No. DE-FG02-97ER-41036.

\section{References}

1. M. Kobayashi and T. Maskawa, Prog. Theor. Phys. 49, 652 (1973).

2. G. 'tHooft, Phys. Rev. Lett. 37, 8 (1976) R. Jackiw and C. Rebbi, Phys. Rev. Lett. 37, 172 (1976) C.G. Callan, R.F. Dashen, and D.J. Gross, Phys. Lett. B63, 334 (1976).

3. M.V. Romalis, W.C. Griffith and E.N. Fortson, hep-ex/0012001.

4. P.H. Frampton, S.L. Glashow and T. Yoshikawa, Phys. Rev. Lett. 87, 011801 (2001).

5. A.D. Sakharov, JETP Lett. 5, 24 (1967).

6. A. Abashian, et al. (BELLE Collaboration). Phys. Rev. Lett. 86, 2509 (2001).

B. Aubert, et al. (BABAR Collaboration). Phys. Rev. Lett. 86, 2515 (2001).

7. S.M. Barr, Phys. Lett. B448, 41 (1999);

See also: S.M. Barr and A.Zee, Phys. Rev. Lett. 55, 2253 (1985); S.M. Barr, Phys. Rev. D34, 1567 (1986); S.M. Barr and E.M. Freire Phys. Rev. D41, 2129 (1990). 
8. P.F. Frampton and T.W. Kephart, Phys. Rev. Lett. 66, 1666 (1991), P.H. Frampton and M. Harada, Phys. Rev. D59, 036004 (1999).

9. D. Bowser-Chao, D. Chang and W.Y. Keung, Phys. Rev. Lett. 81, 2028 (1998) and Phys. Rev. D59, 035004 (1999).

10. H. Georgi and S.L. Glashow, Phys. Lett. B451, 372 (1999).

11. P.H. Frampton and S.L. Glashow, Phys. Rev. D55, 1691 (1997).

12. A.W. Ackley, P.H. Frampton, B. Kayser and C.N. Leung, Phys. Rev. D50, 3560 (1994).

13. A.J. Buras. hep-ph/9905437.

14. S. Adler et al Phys. Rev. Lett. 84, 3768 (2000) 